\title{
The microbiome in chronic inflammatory airway disease: A threatened species
}

R J Green, ${ }^{1}$ PhD, DSc; A van Niekerk, ${ }^{2}$ MB ChB, MMed; A C Jeevarathnum, ${ }^{1}$ MB BCh, FCPaed (SA), MMed, Cert Pulmonology (SA) Paed; C Feldman, ${ }^{3} \mathrm{PhD}$, DSc; G A Richards, ${ }^{3} \mathrm{MB} \mathrm{ChB}, \mathrm{FCP}(\mathrm{SA}), \mathrm{PhD}$; on behalf of the South African Allergic Rhinitis Working Group

${ }^{1}$ Department of Paediatrics and Child Health, Faculty of Health Sciences, University of Pretoria, South Africa

${ }^{2}$ Department of Paediatrics and Child Health, Faculty of Health Sciences, University of Pretoria; and Nectare Clinton Clinic, Johannesburg, South Africa

${ }^{3}$ Department of Medicine, Faculty of Health Sciences, University of the Witwatersrand, Johannesburg, South Africa

Corresponding author: R J Green (robin.green@up.ac.za)

The human body is exposed to a multitude of microbes and infectious organisms throughout life. Many of these organisms colonise the skin, gastrointestinal tract (GIT) and airway. We now recognise that this colonisation includes the lower airway, previously thought to be sterile. These colonising organisms play an important role in disease prevention, including an array of chronic inflammatory conditions that are unrelated to infectious diseases. However, new evidence of immune dysregulation suggests that early colonisation, especially of the GIT and airway, by pathogenic micro-organisms, has deleterious effects that may contribute to the potential to induce chronic inflammation in young children, which may only express itself in adult life.

S Afr Med J 2016;106(8):779-781. DOI:10.7196/SAMJ.2016.v106i8.11159

Every organ system of the human body is alive with micro-organisms, i.e. the microbiome. This constitutes a group of microbial organisms that inhabit every system of the body and consists of microbial species and their associated genomes living on, and in, humans. Although there are multiple distinct microbiomes found at distinct sites and there is individual variation, the composition at any site is broadly similar in health. The microbiome interacts extensively with the host and outnumbers human gene content 100-fold. Among its functions are to afford mucosal protection to allergens and infecting organisms, ${ }^{[1]}$ which also contribute to metabolic capacity and drive immune development. Disturbances in the microbiome are associated with many inflammatory and autoimmune diseases.

The human body is exposed to a multitude of microbes and infectious organisms throughout life. Many of these organisms colonise the skin, gastrointestinal tract (GIT) and airway. We now recognise that this colonisation includes the lower airway, previously thought to be sterile. These colonising organisms play an important role in disease prevention, including an array of chronic inflammatory conditions that are unrelated to infectious diseases. However, new evidence of immune dysregulation suggests that early colonisation, especially of the GIT and airway, by pathogenic micro-organisms, has deleterious effects that may contribute to the potential to induce chronic inflammation in young children, which may only express itself in adult life.

Atopic individuals, who have an underlying allergic condition, have frequent disease exacerbations, mostly produced by infectious organisms, viruses and bacteria, depending on the specific condition.

A new concept is emerging in the aetiology and therefore the prevention of many chronic diseases (including allergy, metabolic syndrome and other chronic inflammatory conditions) through a new understanding of the importance of the interaction of the body with the human microbiome early in life.

In the aetiology of allergic conditions it is now well known that allergic predisposition is conveyed on a genetically at-risk young child by the interaction of perturbation of the child's gastrointestinal microbiome, lack of allergen exposure early in life (and especially foods in the young gut) and absence of immune-modulatory support. ${ }^{[2]}$ This model is well developed in the context of food allergy and atopic dermatitis, but there is a paucity of data on these associations with airway allergic diseases. ${ }^{[3]}$

Therefore, although it seems likely that an allergic diathesis developing in a young child will ultimately lead to allergic rhinitis and atopic asthma through the concept of the allergic march, there must be other or additional mechanisms to explain the onset of these airway inflammatory diseases. ${ }^{[3]}$ The local airway microbiome is important in this regard and changes here may well spell the onset of disease.

Since the description of the hygiene hypothesis by $\operatorname{Strachan}^{[4]}$ in 1989 , there has been significant work in this domain. While the original hypothesis derived from his study showed that a child's risk of developing allergic rhinitis (hay fever) was inversely related to the number of older siblings in the family, and the suggestion that microbial exposure early in life protects against allergic rhinitis, eczema and asthma, the term has evolved. More appropriate terms may be the microbial hypothesis (avoiding an overemphasis on cleanliness), old-friends hypothesis (implying that microbes that were beneficial for immune system development have been eliminated or replaced) and probably most correctly the biodiversity hypothesis or biome depletion. ${ }^{[5]}$ The real problem is that many chronic inflammatory diseases, including allergy, are associated with both a replacement and a depletion of the normal microbiome (Fig. 1).

The normal microbiome (both diversity and content) is important in its interaction with pattern recognition receptors on epithelial cells, which, in turn, regulate the relationship between pro- and antiinflammatory cytokines, chemokines and mediators. ${ }^{[6]}$

Finally, once a chronic inflammatory airway disease has developed, there is an ongoing and important relationship with the local microbiome in disease exacerbations.

Organisms are more numerous in the upper-respiratory than the lowerrespiratory tract. Healthy subjects demonstrate a diverse microbiome, which often includes potential pathogens such as Pseudomonas aeruginosa, Staphylococcus aureus and various streptococcal species. When infection ensues, the microbiome is disrupted, causing community collapse with loss of diversity and overgrowth of pathogenic species. ${ }^{[1]}$ 
Despite the seemingly unrelated association between the early gut and the respiratory tract, there is a certain cross-talk between the microbiome in the GIT and the airway through a gut-respiratory tract axis so that changes in the GIT microbiome create physiological changes in the airway (Fig. 2). ${ }^{[7]}$

\section{Chronic airway disease and bacterial colonisation of the respiratory tract}

There is now clear evidence that colonisation of the nasopharynx in the first month of life by Streptococcus pneumoniae, Haemophilus influenzae and Moraxella catarrhalis is positively associated with recurrent wheeze, followed by asthma by 5 years of age. ${ }^{[8]}$ It may be true that the origin of allergic rhinitis has a similar link

\section{The bacterial}

\section{microbiome in chronic rhinosinusitis}

Once a chronic allergic and inflammatory diathesis is established in the upper-respira- tory tract, both the nose and sinuses become inflamed and symptoms ensue. These sites also harbour a microbiome that is intimately involved in the allergic disease process, both chronically and during disease exacerbations. It is difficult to call this the normal microbiome, as the airway is already ill. It is a resident microbiome. Conventional culture of airway secretions may reveal the usual bacterial species, including $S$. aureus (and coagulase-negative staphylococci), streptococci spp., H. influenzae and anaerobes (Prevotella and Peptostreptococcus). However, there is significant variability between studies. $^{[6]}$

New techinques employing the 16S rRNA gene wide sequencing have uncovered a population of bacteria that are not found on conventional culture. However, different studies reveal different organisms. Stephenson et al. ${ }^{\left[{ }^{[]}\right.}$ identified anaerobic bacteria (Diaphorobacter, Peptoniphilus) in $78 \%$ and $72 \%$ of chronic rhinosinusitis cases, respectively, but not in controls, and S. aureus was found in half of the chronic rhinosinusitis cases, while it was detected in all controls. ${ }^{[9]}$

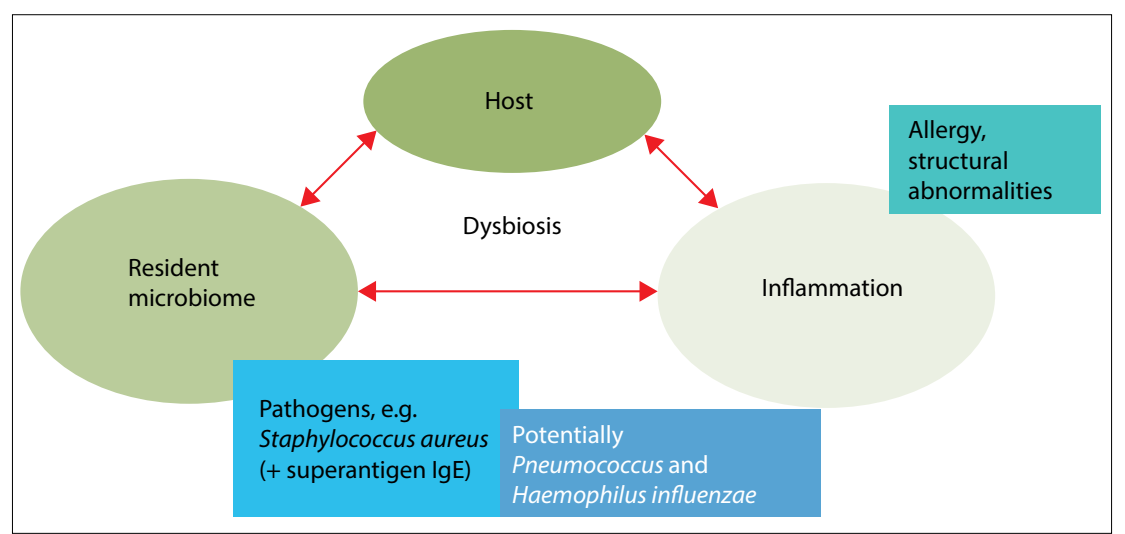

Fig. 1. The microbiome-inflammation interaction - microbiome depletion in the upper-respiratory tract and disease.

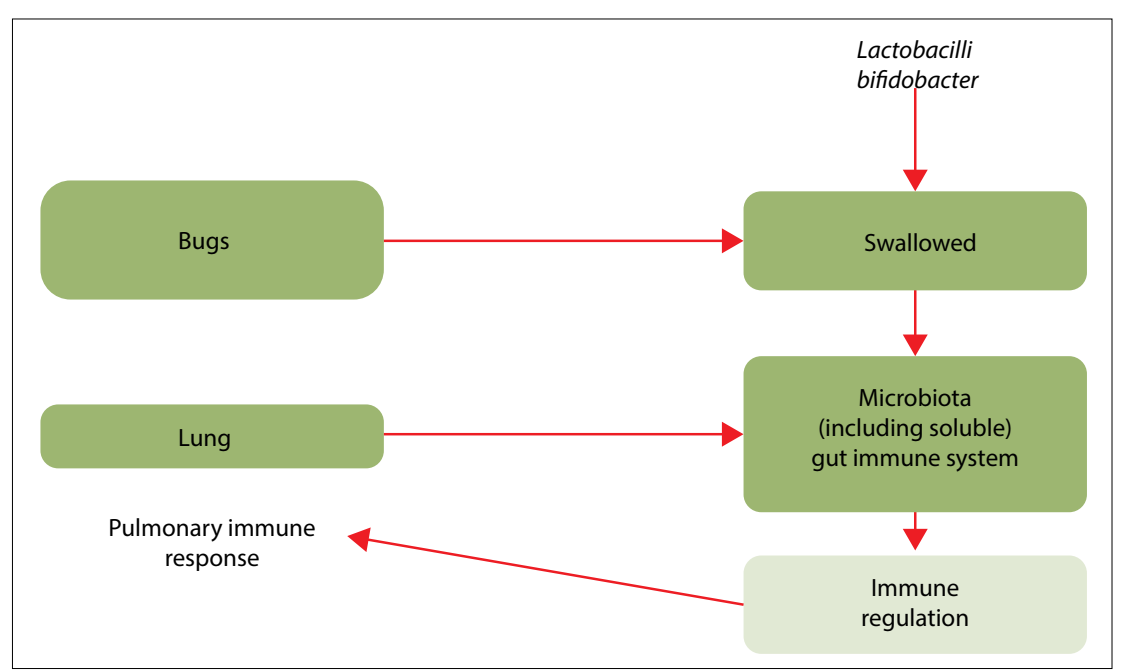

Fig. 2. The gut-respiratory tract axis of allergy development.
Another study, however, revealed Pseudomonas, Citrobacter, Haemophilus propionibacterium, Staphylococcus and Streptococcus as the resident microbiomes. ${ }^{[10]}$

There are a number of confounders in these studies, however, which may in part explain different organism identification. These confounders include antibiotic use, steroid use, sampling method, exacerbations, variability in constitutive epithelial host-defense molecule secretion based on the region of the nose and sinuses sampled, and lastly the associated comorbidities. ${ }^{[1]}$

It seems that an explanation for these study differences lies therein that the microbiome is individual specific, which may be one of the explanations for difficulty in therapeutic attempts.

\section{The fungal and viral microbiome in chronic rhinosinusitis}

Just as there is a resident bacterial microbiome in chronic rhinosinusitis, there is also a fungal microbiome. Species, including Candida, Aspergillus and Mucor, differ in studies and may even be found in asymptomatic control subjects. ${ }^{[12]}$

Employing multiplex polymerase chain reaction for respiratory viruses, viral nucleic acid sequences have been found in $64 \%$ of sinus scrapings and $50 \%$ of nasal lavage samples in a chronic rhinosinusitis group of patients. ${ }^{[13]}$ These were significantly higher than in controls (30\% and $14 \%$ in scraping and lavage, respectively) and rhinovirus was the most frequently detected virus. ${ }^{[13]}$

\section{Biofillm}

Many of the early microbiological studies of bacteria, including their discovery and characterisation, were performed with planktonic bacteria, which are characterised as individual free-living (free-swimming) bacteria. ${ }^{[14]}$ Even currently, standard laboratory culture techniques use planktonic bacteria that grow rapidly in nutrient-rich media. However, with the use of modern molecular techniques, it has become apparent that there are large populations of organisms in the human host that cannot be cultured by these standard techniques, as these organisms have adapted to a different type of growth ${ }^{[14]}$ Most bacteria exist in complex, tightknit colonies or communities often strongly adherent to surfaces; it has been reported that $<1 \%$ of bacteria exist in planktonic form and that $\geq 99 \%$ of bacteria live in these types of biofilms. ${ }^{[1-16]}$

Biofilms comprise highly structured communities of bacteria that exist within an extracellular matrix that consists of polysaccharides, nucleic acids and proteins, the so-called extracellular polymeric substances. ${ }^{[15,16]}$ 
The formation of mature biofilms occurs in stages, proceeding through a number of well-characterised steps that are regulated by the bacterial quorum sensing system. ${ }^{[15-17]}$ The latter involves communication between the bacterial cells through the release of small protein pheromones that control bacterial gene expression and therefore co-ordinate their behaviour. ${ }^{[18]}$ Many bacteria, particularly those associated with chronic infection, including S. pneumoniae, $P$. aeruginosa, $H$. influenzae, S. aureus and Escherichia coli, are known to produce biofilm. ${ }^{[17-19]}$

The characteristics of the micro-organisms in biofilm differ greatly from those of planktonic bacteria. ${ }^{[14]}$ Bacteria within biofilms undergo changes in their expression of virulence factors and/or surface molecules, their utilisation of nutrients, and their cell turnover rates. ${ }^{[19]}$ Also, very importantly, there is an increase in antibiotic resistance in bacterial biofilms. Bacterial biofilms may be up to 1000 times more resistant to antibiotics than planktonic bacteria, which are genetically identical. ${ }^{[15]}$ The mechanism of antibiotic resistance appears to be multifactorial, including factors such as impeded penetration of antibiotics into the biofilm. ${ }^{[19]}$ However, one important mechanism relates to a slow metabolic rate and turnover of bacteria deep within the nutrient-depleted zone of the biofilm so that these micro-organisms are less susceptible to antibiotics that target cell wall synthesis, which are much more active against bacteria that turn over rapidly. ${ }^{[1,19]}$

Biofilm bacteria are also more resistant to host defences, which frequently results in both persistence of the biofilms and ongoing surrounding inflammation. ${ }^{[15]}$ We are beginning to understand the important role of bacterial biofilms in a number of infections, including chronic lower-airway infections, such as cystic fibrosis, bronchiectasis and chronic obstructive pulmonary disease; device-related infections; and various otolaryngology conditions, including chronic rhinosinusitis, among many others, which are extremely difficult to treat. ${ }^{[14-16,19]}$

\section{Current status of probiotic use in chronic airway disease management}

It is theoretically possible that restoration of the microbiome could increase resistance to infection and decrease allergic responses. In this regard, numerous studies have been performed, most relying on the link between the gut and the respiratory tract, the so-called gutrespiratory tract axis. Unfortunately, these studies have generally been small, have used multiple different probiotics in different doses, and have utilised different administration schedules. A recent systematic review of the literature showed seven randomised, placebo-controlled trials involving the use of different probiotics. Two studies $(N=170)$ specifically evaluated quality of life (QOL), frequency of symptoms, and level of bother, and found a statistically significant improvement. Although there was a decrease in episodes of rhinitis per year, there was no change in blood or immunological parameters and no effect on time free from rhinitis episodes or mean duration of episodes. ${ }^{[20]}$

Similarly, a more recent systematic review and meta-analysis involving MEDLINE, Embase and Cochrane Library databases, found 21 double-blind randomised controlled trials and two randomised crossover studies ( $N=1919)$ involving multiple probiotics, populations and outcomes. ${ }^{[21]}$ Seventeen of these showed significant clinical benefit in one or more outcome measures; however, six showed no benefit. Among the trials eligible for meta-analysis, the rhinitis QOL score improved significantly, but there was no effect on total symptom score or IgE. Their conclusion was 'that probiotics may be beneficial in improving symptoms and QOL in patients with allergic rhinitis; however, current evidence remains limited due to study heterogeneity and variable outcome measures. Additional high-quality studies are needed to establish appropriate recommendations. ${ }^{[21]}$

Some unanswered questions therefore arise from these studies and this replacement concept. These include what mixture of probiotics is best, what the timing of administration should be, and where best the probiotics should be sited (the gut or directly into the nose or sinuses).

As there is good evidence for efficacy (microbial burden change and inflammatory response modification) in mouse models for topical application of probiotics, this model needs urgent human studies.

\section{Conclusion}

The microbiome in the upper-respiratory tract is a dynamic organ. Dysbiosis is easy to produce in many disease states, and with the use of many drug therapies, including antibiotics and nasal steroids, the upper-respiratory tract is susceptible to significant pertubations and exacerbations.

As studies demonstrating that restoring the microbiome in the nose and sinuses are currently not effective, it seems prudent to try to protect the resident or normal microbiome at all costs.

Both the microbiome of the young gut and that of the upperrespiratory tract early in life are vital to disease prevention, as when dysbiosis and depletion occur, chronic inflammatory disease ensues.

As a society, and as a scientific community, we need to look carefully at the many strategies we currently allow that interfere with the health of this important human asset, the normal microbiome. Once gone, it is almost certainly too late to retrieve it.

Conflict of interest. The South African Allergic Rhinitis Working Group is an independent organisation. An annual meeting is supported by an unrestricted educational grant from Aspen Pharma.

The South African Allergic Rhinitis Working Group: M Hockman (chair), R Friedman (deputy chair), H Lewis, M Davis, C Els, S Motakef, S Bouwer, P Crossland, A McCulloch, P Pio, M Gill, J Coetzee, K Kalideen, C Lodder.

1. Cope EK, Lynch SV. Novel microbiome-based therapeutics for chronic rhinosinusitis. Curr Allergy Asthma Rep 2015;15(3):504. DOI:10.1007/s11882-014-0504-y

2. Von Mutius E. Allergies, infections and the hygiene hypothesis: The epidemiological evidence. Immunobiolog 2007;212(6):433-439.

3. Holt PG, Strickland DH, Hales BJ, Sly PD. Defective respiratory tract immune surveillance in asthma: A primary casual factor in disease onset and progression. Chest 2014;145(2):370-378. DOI:10.1378 chest.13-1341

4. Strachan DP. Hay fever, hygiene and household size. BMJ 1989;299(6710):1259-1260. DOI:10.1136/ bmj. 299.6710 .1259

. Green RJ. The interaction of respiratory allergies and 'infection'. Curr Allergy Clin Immunol 2015;28:84-87. 6. Mahdavinia M, Keshavarzian A, Tobin MC, Landay AL, Schleimer RP. A comprehensive review of the nasal microbiome in chronic rhinosinusitis (CRS). Clin Exp Allergy 2016;46(1):21-41. DOI:10.1111/cea.12666

7. Bassis CM, Erb-Downward JR, Dickson RP, et al. Analysis of the upper respiratory tract microbiotas Bassis CM, Erb-Downward JR, Dickson RP, et al. Analysis of the upper respiratory tract microbiotas
as the source of the lung and gastric microbiotas in healthy individuals. MBio 2015;6(2):e00037. as the source of the lung an
DOI:10.1128/mBio.00037-15

DOI:10.1128/mBio.00037-15
8. Bisgaard H, Hermansen MN, Buchvald F, et al. Childhood asthma after bacterial colonization of the airway in neonates. N Engl J Med 2007;357(15):1487-1495. DOI:10.1056/NEJMoa052632

9. Stephenson MF, Mfuna L, Dowd SE, et al. Molecular characterization of the polymicrobial flora in chronic rhinosinusitis. J Otolaryngol Head Neck Surg 2010;39(2):182-187.

0. Stressmann FA, Rogers GB, Chan SW, et al. Characterization of bacterial community diversity in chronic rhinosinusitis infections using novel culture-independent techniques. Am J Rhinol Allergy 2011;25(4):e133-e140. DOI:10.2500/ajra.2011.25.3628

11. Ramakrishnan VR, Hauser LJ, Feazel LM, Ir D, Robertson CE, Frank DN. Sinus microbiota varies among chronic rhinosinusitis phenotypes and predicts surgical outcome. J Allergy Clin Immunol 2015;136(2):334-342. DOI:10.1016/j.jaci.2015.02.008

12. Cleland EJ, Bassiouni A, Boase S, Dowd S, Vreugde S, Wormald PJ. The fungal microbiome in chronic rhinosinusitis: Richness, diversity, postoperative changes and patient outcomes. Int Forum Allergy rhinosinusitis: Richness, diversity, postoperative
Rhinol 2014;44(4):259-265. DOI:10.1002/alr.21297

13. Cho GS, Moon BJ, Lee BJ, et al. High rates of detection of respiratory viruses in the nasal washes and mucosae of patients with chronic rhinosinusitis. Clin Microbiol 2013;51(3):979-984. DOI: 10.1128/JCM.02806-12

14. Pragman AA, Berger JP, Williams BJ. Understanding persistent bacterial lung infections: Clinical implications informed by the biology of the microbiota and biofilms. Clin Pulm Med 2016;23(2):57-66. DOI: $10.1097 / \mathrm{cpm} .0000000000000108$

15. Post JC, Hiller NL, Nistico L, et al. The role of biofilms in otolaryngologic infections: Update 2007. Cur Opin Otolaryngol Head Neck Surg 2007;15(5):347-351.

6. Al-Mutairi D, Kilty SJ. Bacterial biofilms and the pathophysiology of chronic rhinosinusitis. Curr Opin Allergy Clin Immunol 2011;11(1):18-23. DOI:10.1097/ACI.0b013e3283423376

7. Suntharalingam P, Cvitkovitch DG. Quorum sensing in streptococcal biofilm formation. Trends Microbiol 2005;13(1):3-6. DOI:10.1016/j.tim.2004.11.009

18. Moscoso M, García E, López R. Pneumococcal biofilms. Int Microbiol 2009;12:77-85.

19. Hall-Stoodley L, Stoodley P. Evolving concepts in biofilm infections. Cell Microbiol 2009;11(7):1034-1043. DOI:10.1111/j.1462-5822.

20. Das RR, Singh M, Shafiq N. Probiotics in treatment of allergic rhinitis. World Allergy Organ J 2010;3(9):239-244. DOI:10.1097/WOX.0b013e3181f234d4

21. Zajac AE, Adams AS, Turner JH. A systematic review and meta-analysis of probiotics for the treatment of allergic rhinitis. Int Forum Allergy Rhinol 2015;5(6):524-532. DOI:10.1002/alr.21492 